\title{
Efficient delivery of docetaxel for the treatment of brain tumors by cyclic RGD-tagged polymeric micelles
}

\author{
AI-JUN LI ${ }^{1}$, YUE-HUA ZHENG ${ }^{1}$, GUO-DONG LIU ${ }^{2}$, WEI-SHENG LIU ${ }^{1}$, \\ PEI-CHENG CAO ${ }^{1}$ and ZHEN-FU BU ${ }^{1}$ \\ ${ }^{1}$ Department of Neurosurgery, Weifang People's Hospital, Weifang, Shandong 261021; ${ }^{2}$ Department of Neurosurgery, \\ Second Affiliated Hospital of Soochow University, Suzhou, Jiangsu 215004, P.R. China
}

Received March 31, 2014; Accepted July 25, 2014

DOI: $10.3892 / \mathrm{mmr} .2014 .3017$

\begin{abstract}
The treatment of glioblastoma, and other types of brain cancer, is limited due to the poor transport of drugs across the blood brain barrier and poor penetration of the blood-brain-tumor barrier. In the present study, cyclic Arginine-Glycine-Aspartic acid-D-Tyrosine-Lysine [c(RGDyK)], that has a high binding affinity to integrin $\alpha v \beta 3$ receptors, that are overexpressed in glioblastoma cancers, was employed as a novel approach to target cancer by delivering therapeutic molecules intracellularly. The c(RGDyK)/docetaxel polylactic acid-polyethylene glycol (DTX-PLA-PEG) micelle was prepared and characterized for various in vitro and in vivo parameters. The specific binding affinity of the Arginine-Glycine-Aspartic acid (RGD) micelles, to the integrin receptor, enhanced the intracellular accumulation of DTX, and markedly increased its cytotoxic efficacy. The effect of microtubule stabilization was evident in the inhibition of glioma spheroid volume. Upon intravenous administration, c(RGDyK)/DTX-PLA-PEG showed enhanced accumulation in brain tumor tissues through active internalization, whereas non-targeted micelles showed limited transport ability. Furthermore, RGD-linked micelles showed marked anti-glioma activity in U87MG malignant glioma tumor xenografts, and significantly suppressed the growth of tumors without signs of systemic toxicity. In conclusion, the results of the present study suggest that ligand-mediated drug delivery may improve the efficacy of brain cancer chemotherapy.
\end{abstract}

\section{Introduction}

Glioblastoma multiforme (GBM) is one of the most common and aggressive forms of brain tumor, accounting for $50-60 \%$

Correspondence to: Dr Wei-Sheng Liu, Department of Neurosurgery, Weifang People's Hospital, 151 Guangwen Street, Weifang, Shandong 261041, P.R. China

E-mail:drliuws@outlook.com

Key words: c(RGDyK), polymeric micelles, docetaxel, glioblastoma, brain cancer of all brain cancers in humans, and is associated with a low median survival rate (1). GBM is generally characterized by high lethality, invasiveness, excessive growth, and a poor prognosis $(2,3)$. The current gold-standard treatment strategy for brain tumors is comprised of radiation therapy, surgical removal, and chemotherapy. However, difficulties in surgical excision, and the severe adverse effects associated with irradiation and chemotherapy, hinder these approaches $(4,5)$. Furthermore, the advanced chemotherapy regimen has been reported as having limited or no impact on brain tumors due to the poor penetration profile of the drug through the highly variable, heterogeneous blood brain barrier (BBB) (6). In addition, a protective blood-brain tumor-barrier (BBTB) has been shown to form around tumor cells alongside the progression of GBM, which regulates the entry of small molecules (7). Therefore, any approach that can overcome the tight extracellular junctions of both the BBB and BBTB would be a promising treatment solution.

Nanotechnology may be a prospective method to overcome the BBB and BBTB. Polymeric nanoparticles (NP), or polymeric micelles, have drawn significant attention due to their ability to surpass the physiological barriers and improve the delivery of therapeutic agents into the brain $(8,9)$. The enhanced permeability and retention effect is the main factor that allows the preferential accumulation of NP in the tumor fenestrations. However, limited penetrability, and non-uniform distribution into other body parts, impairs the performance of simple carrier-mediated delivery systems (10). Receptor-mediated active targeting moiety-tagged NPs may be a feasible approach in the translocation of the carriers. Cyclic Arginine-Glycine-Aspartic acid (cRGD) has been selected as a targeting moiety to promote the biological interactions between the delivery carrier and the overexpressed receptor in the tumor cells (11). Specifically, $\alpha v \beta 3$ and $\alpha v \beta 5$ integrins, which are over-expressed in tumor endothelial and GBM cells, have been shown to have a selective affinity to cRGD (12). Chen et al (13) successfully demonstrated the in vivo imaging of GBM, with the aid of an RGD-containing probe. Furthermore, an RGD-iron oxide NP conjugate has been developed, and shown to enhance the targeting efficiency in brain tumors (14).

Docetaxel (DTX) is an anti-mitotic taxane drug, considered to be one of the most effective drugs against brain tumors (15). The present study developed polylactic 
acid-polyethylene glycol (PLA-PEG)-based long circulating, DTX-loaded, cyclic RGD-attached polymeric micelles [DTX-PLA-PEG/c(RGDyK)]. These micelles could prolong the blood circulation time of the carriers, and specifically target the integrin receptors known to be over-expressed in brain tumors. The PEG provided an anti-fouling property and extended the circulation time of the carriers, whilst the cRGD specifically targeted the tumor cells. The aim of the present study was to develop a functional NP system which could be transported across the physiological barriers of the brain, and effectively deliver the therapeutic load. Pharmacokinetic and biodistribution studies were performed to quantify the amount of drug accumulated in the brain tissue. The antitumor efficacy of the system was determined using a U87MG bearing xenograft tumor model, by measuring the tumor volume and survival rate index. The study investigated whether cRGD-linked polymeric micelles can effectively penetrate the brain tumor and exhibit a potent antitumor effect.

\section{Materials and methods}

Materials. The materials used for the subsequent experiements were provided by the following suppliers. The methoxyl poly(ethylene glycol) (mPEG-OH, $2 \mathrm{kDa}$ ) and the maleimide-poly(ethylene glycol) (mal-PEG-OH, $3.5 \mathrm{kDa}$ ) were obtained from Sigma-Aldrich (Hong Kong, China). The benzotriazole-N,N,N,N'-tetramethyl-uronium-hexafluorophosphate was from American Bioanalytical Inc. (Natick, MA, USA). Benzotriazol-1-yl-oxytripyrrolidinophosphonium hexafluorophosphate was obtained from GL Biochem Ltd (Shanghai, China). Diisopropylethylamine and H-Gly-2-Chlorotrityl resin were both supplied by Fluka (Sigma-Aldrich). 3,3'-dithiodipronic acid and D,L-dithiotheriol (DTT) were purchased from Aladdin Reagents Co. (Shanghai, China). Cyclo[RGDfK(CX-)] (cRGD peptide, $\mathrm{X}=6$-aminocaproic acid:-Acp) was purchased from Peptide Institute Inc. (Osaka, Japan). DTX was procured from Sigma-Aldrich (China).

Synthesis of RGD-PLA-PEG polymer. The synthesis of PLA-PEG was performed as reported by previous methods (16). The PLA-PEG was conjugated with the thiolated $\mathrm{c}(\mathrm{RGDyK})$ to form $\mathrm{c}(\mathrm{RGDyK})-\mathrm{PLA}-\mathrm{PEG}$ as previously reported (17). Briefly, 3,3'-dithiodipronic acid was activated by dicyclohexylcarbodiimide (DCC) and N-hydroxysuccinimide (NHS) and the resulting active ester was reacted with $\mathrm{c}(\mathrm{RGDyK})$. The obtained product was reduced with DTT to obtain the thiolated cRGD peptide. To conjugate the peptide with PLA-PEG, mal-PLA-PEG was dissolved in acetonitrile (ACN) and a thin-film was formed by rotary evaporation. Phosphate-buffered saline (PBS-hydrated PLA-PEG was added to the $\mathrm{c}(\mathrm{RGDyK})-\mathrm{SH}$ and stirred overnight. The resulting product was extracted and characterized by nuclear magnetic resonance. The polymers were characterized using ${ }^{1} \mathrm{H}$ nuclear magnetic resonance (NMR; solvents, $\mathrm{CDCl}_{3}$, $\mathrm{D}_{2} \mathrm{O}$ and deuterated-dimethyl sulfoxide (DMSO); temperature, $25^{\circ} \mathrm{C}$ ). The NMR spectra were recorded using a JEOL Alpha 500 spectrometer (500 MHz; Jeol, Ltd., Tokyo, Japan).

Preparation of DTX-loaded micelles. The DTX-loaded polymeric micelles were formed using a thin-film hydration technique. Briefly, $10 \mathrm{mg}$ of PLA-PEG and $5 \mathrm{mg}$ of DTX were dissolved in $5 \mathrm{ml}$ of ACN. The mixture was rotary-evaporated and the thin-film was subsequently hydrated with PBS. For the c(RGDyK)-tagged micelles, 5\% w/w c(RGDyK)-PLA-PEG was mixed with 95\% DTX-PLA-PEG. The resulting micelles were evaluated for drug loading and dynamic light scattering (DLS) characteristics using a Malvern Zetasizer (Malvern Instruments Ltd., Malvern, UK).

Particle size distribution and zeta potential. The micellar solutions were suitably diluted, in order to analyze the particle size distribution and zeta potential, using the DLS method. A Malvern Zetasizer was used to determine the DLS characteristics. All measurements were performed at a fixed angle of $90^{\circ}$ at $25^{\circ} \mathrm{C}$. The results were expressed as the size \pm standard deviation.

DTX loading efficiency. The loading efficiency of the carrier was calculated from the total amount of drug added, versus the amount of drug entrapped within the nanoparticles. Briefly, DTX-PLA-PEG (DPP), and RGD/DTX-PLA-PEG (RDPP) were filtered using an Amicon ${ }^{\circledR}$ centrifugal filter (Millipore, Billerica, MA, USA) at $550 \mathrm{x}$ g for $10 \mathrm{~min}$. The filtrate was then analyzed for unentrapped drugs by high performance liquid chromatography (HPLC). The mobile phase (acetonitrile : water, 60:40, $\mathrm{pH} 3.5$ ) was set at $1 \mathrm{ml} / \mathrm{min}$ with an absorbance of $254 \mathrm{~nm}$.

In vitro release study. The in vitro release of DTX from both PLA-PEG and RGD/PLA-PEG was evaluated by dialysis. A total of $1 \mathrm{ml}$ of NP dispersions were placed in a dialysis bag (3000 MW cutoff; Spectra/Por ${ }^{\circledR}$, Spectrum Laboratories, Inc., Rancho Dominguez, CA, USA) and both the borders were sealed with a dialysis clip. The dialysis bag was incubated in $30 \mathrm{ml}$ release media (PBS, pH 7.4), containing 1\% Tween ${ }^{\circledR} 80$ as a solubilizer. The bag was placed in an automated shaker maintained at $2 \times \mathrm{g}$ and $37^{\circ} \mathrm{C}$. At a specified time $(1,2,4,8$, $12,24,48,72,96,120,144$ and $168 \mathrm{~h})$, the release media was collected and replaced with an equal amount of fresh media. The amount of released drug was quantified by HPLC.

Cytotoxicity assay. U87MG human malignant glioma and 9L rat gliosarcoma cells (ATCC, Manassas, VA, USA) were cultured in normal RPMI-1640 media supplemented with $10 \%$ fetal bovine serum and $1 \%$ penicillin-streptomycin in a $5 \% \mathrm{CO}_{2}$ and $95 \%$ humidified atmosphere. The viability of the cells was determined by MTT assay (Sigma-Aldrich). Briefly, the cells were seeded into a 96-well plate, at a seeding density of $1 \times 10^{4}$ cells, and incubated for $24 \mathrm{~h}$. The following day, the media was refreshed and various concentrations of free DTX, DPP or RDPP were exposed to the cells followed by 24 and $48 \mathrm{~h}$ incubations. The cells were then washed and treated with MTT solution (5 mg/ml in serum-free media) and incubated for $3 \mathrm{~h}$. The purple blue formazan crystals were extracted by the addition of DMSO and the absorbance was detected using a plate reader (Multiskan Ascent, Labsystems SA, Cergy-Pontoise, France) at $570 \mathrm{~nm}$.

Cellular uptake study. A cellular uptake study was performed to investigate the kinetics of drug internalization. A total of 
Table I. Physicochemical characterization of micelles.

\begin{tabular}{lrcccr}
\hline Micelle & Size $(\mathrm{nm})$ & PDI & Charge (m) & EE $(\%)$ & DLS (\%) \\
\hline DTX-PLA-PEG & $90.5 \pm 2.5$ & $0.158 \pm 0.002$ & $-28.6 \pm 1.23$ & $94.2 \pm 2.6$ & $30.26 \pm 3.42$ \\
DTX-PLA-PEG/c(RGDyK) & $118.4 \pm 1.6$ & $0.234 \pm 0.003$ & $-22.4 \pm 1.34$ & $90.4 \pm 3.6$ & $28.65 \pm 1.46$ \\
\hline
\end{tabular}

DTX-PLA-PEG, docetaxel polylactic acid-polyethylene glycol; c(RGDyK), cyclic Arginine-Glycine-Aspartic acid-D-Tyrosine-Lysine; PDI, polydispersity index; EE, entrapment efficiency; DLS, dynamic light scattering.

$1 \times 10^{6}$ U87MG and 9L cells were seeded in a 6 -well plate and allowed to adhere for $24 \mathrm{~h}$. The following formulations, free DTX, DPP or RDPP (DTX equivalent $20 \mu \mathrm{g} / \mathrm{ml}$ ) were incubated with the cells for 1-4 h. Following the incubation, the media was removed and the cells were washed twice with PBS. An aliquot of lysis buffer was added to lyse the cells, followed by centrifugation at $500 \mathrm{x}$ g for $10 \mathrm{~min}$. The lysis buffer (Sigma-Aldrich) consisted of $50 \mathrm{mmol} / 1 \mathrm{Tris}$, $150 \mathrm{mmol} / 1 \mathrm{NaCl}, 1 \%$ Triton X-100, $1 \%$ sodium deoxycholate, $0.1 \%$ sodium dodecyl sulfate, $1 \mathrm{mmol} / 1$ sodium orthovanadate, $1 \mathrm{mmol} / 1$ sodium fluoride, $1 \mathrm{mmol} / 1$ EDTA and $1 \mathrm{mmol} / 1$ phenylmethylsulfonyl fluoride. The supernatant was collected and analyzed by HPLC. The percentage of cellular uptake of DTX from the various formulations was estimated by normalizing to the initial DTX concentration administered.

Cell cycle analysis. U87MG and 9L cells were seeded in 6 -well plates at a density of $3 \times 10^{5}$ cells/well with RPMI-1640 media for $24 \mathrm{~h}$. The cells were then exposed to various doses of free DTX, DPP or RDPP and further incubated for $24 \mathrm{~h}$. Following incubation the cells were washed and further incubated with propidium iodide $(20 \mu \mathrm{g} / \mathrm{ml})$ for $30 \mathrm{~min}$ The DNA content was measured for 10,000 events for each sample by flow cytometry using a BD FACS Atira II (BD Biosciences, Franklin Lakes, NJ, USA). The data were plotted using Cell Quest $^{\mathrm{TM}}$ (BD Biosciences) software.

Analysis of glioma spheroids in vitro. The inhibitory effect of various formulations on the growth of glioma spheroids was investigated. U87MG tumor spheroids were incubated for a period of seven days, after which the media was refreshed, prior to the application of the formulations. The glioma cells, incubated in RPMI-1640 media, were exposed to free DTX, DPP, or RDPP. On days $0,2,4,6$, and 8 , the tumor spheroids were observed under an inverted microscope. The inhibitory effects of the various formulations were calculated using the following formula: $\mathrm{V}=\left(\pi \times \mathrm{d}_{\max } \times \mathrm{d}_{\min }\right) / 6$, where $\mathrm{d}_{\max }$ and $\mathrm{d}_{\min }$ refer to major and minor diameters, respectively. The spheroid tumor volume at day 8 was calculated using the following formula: ratio $\%=\left(\mathrm{V}_{\text {day } 8} / \mathrm{V}_{\text {day } 0}\right) \times 100 . \mathrm{V}_{\text {day } 0}$ is the spheroid volume t day 0 . The sections were observed using a Zeiss Axioscope fluorescence microscope and photographed using a digital AxioCam mRM camera (Zeiss, Oberkochen, Germany).

Antitumor efficacy of $c(R G D y K)-P L A-P E G-D T X$. Nude mice were obtained from the Experimental Animal Center of Soochow University (Suzhou, China) and maintained under regular light and dark conditions with free access to food. The study was approved by the Institutional Animal Care Committee of Soochow University. U87MG tumor bearing xenograft nude mice were developed by subcutaneously injecting $1 \times 10^{6}$ cells into the right flank of the mice. The tumor growth was monitored until it reached $\sim 100-150 \mathrm{~mm}^{3}$. Approximately two weeks after the tumor cell injection, the experiment was started. The mice were randomly divided into five groups ( $n=6 /$ group). Groups I to IV were administered saline, free DTX, DPP or RDPP, and group V was identified as the untreated, control group. A total of $100 \mu \mathrm{l}$ of each formulation (10 mg/kg body weight of DTX) was injected into the mice subcutaneously twice a week for two weeks. The tumor size was measured biweekly using a caliper, and the tumor volume (V) was calculated using the following formula $\mathrm{V}=1 / 2\left[\mathrm{~L} \times(\mathrm{W})^{2}\right]$; where $\mathrm{L}=$ length and $\mathrm{W}=$ width.

Pharmacokinetics and biodistribution. The pharmacokinetics study was performed in seven week old mice ( 20 g). The mice were divided into three groups ( $\mathrm{n}=5 /$ group). Each group was administered with free DTX, DPP or RDPP $(10 \mathrm{mg} / \mathrm{kg}$ DTX) via tail vein injection. The blood samples were collected at $0.25,0.5,1,2,4,6,8,12$, and $24 \mathrm{~h}$. The samples were immediately centrifuged at $800 \mathrm{x} g$ for $15 \mathrm{~min}$ and stored at $-20^{\circ} \mathrm{C}$ until further analysis. To prepare the plasma samples, $100 \mu \mathrm{l}$ of methanol containing $50 \mathrm{ng} / \mathrm{ml}$ of DTX was added to $50 \mu \mathrm{l}$ of plasma. The mixture was vortexed for $30 \mathrm{~min}$ and subsequently centrifuged. The supernatant was collected and analyzed for DTX content by HPLC.

For the biodistribution study, brain tumor-bearing mice were developed as previously described in the above methods. Three weeks after the tumor implantation, the mice were divided into three groups ( $n=14 /$ group). The groups were administered free DTX, DPP or RDPP (10 mg/kg DTX) via tail vein injection. At a specified time point, two mice were sacrificed and their organs were surgically removed and preserved on ice. At $0.5,1,2,4,6,12$ and $24 \mathrm{~h}$, mice were sacrificed by carbon dioxide inhalation in a closed tube, according to the ethical guidelines. The organs were macerated using a high pressure homogenizer $(10,000 \mathrm{x}$ g; Ultra-Turrax T25 Homogenizer; IKA ${ }^{\circledR}$-Werke GmbH \& Co. KG, Staufen, Germany) and the drug levels present in the homogenate of each organ were analyzed by HPLC.

Statistical analysis. Statistical significance was determined using one-way analysis of variance, followed by Tukey's post hoc test. Statistical significance was evaluated using 
A

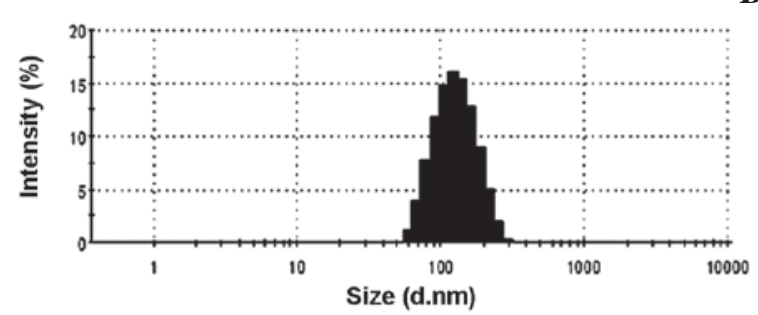

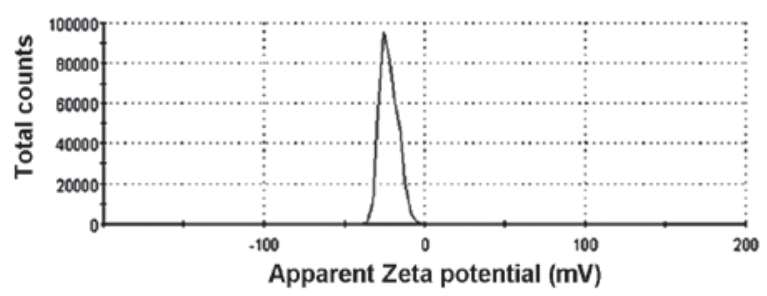

C

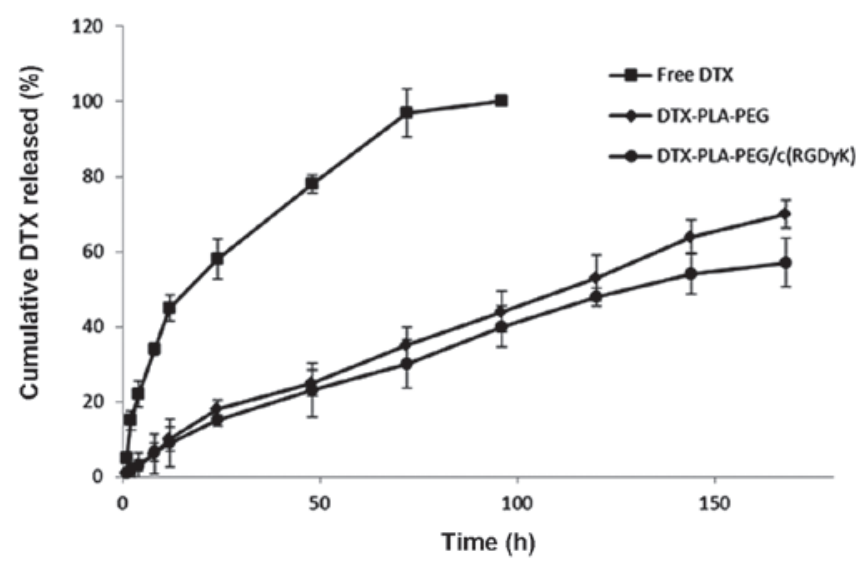

Figure 1. Physicochemical characterization of docetaxel polylactic acid-polyethylene glycoL/cyclic (Arginine-Glycine-Aspartic acid-D-Tyrosine-Lysine) [DTX-PLA-PEG/c(RGDyK)] micelles. (A) Particle size distribution, determined by Zetasizer. (B) Zeta potential analysis. (C) Release kinetics of DTX from DTX-PLA-PEG/c(RGDyK) micelles, DTX-PLA-PEG micelles, or free DTX suspension in phosphate buffered saline (pH 7.4) at $37^{\circ} \mathrm{C}$. The data represents the means \pm standard deviation $(n=3)$. DTX, docetaxel.

SPSS version 5 (SPSS, Inc., Chicago, IL, USA). All values are expressed as the mean \pm standard deviation. $\mathrm{P}<0.05$ was considered to indicate a statistically significant difference.

\section{Results and Discussion}

Characterization of DPP and RDPP micelles. Brain tumors are one of the most aggressive and difficult tumors to treat, partly due to the complexity of the anatomical location. Furthermore, both the BBB and BBTB hamper any therapeutic strategy made to increase the drug accumulation in the brain. An attractive prospect of brain chemotherapy is the use of targeted drug delivery, which target cellular receptors in the brain. Such cellular responsive brain targeting systems are expected to increase the drug concentration in brain tumors. In the present study RGD, which has a high affinity for the $\alpha v \beta 3$ and $\alpha v \beta 5$ integrins in GBM cells, was conjugated with PLA-PEG by a DCC/NHS reaction. DTX was encapsulated within PLA-PEG and RGD/PLA-PEG, resulting in the formation of DPP and RDPP micelles respectively. The mean particle size of DPP was $\sim 90 \mathrm{~nm}$ with a uniform size distribution (polydispersity index $\sim 0.150$ ) (Table 1 ). RGD-linking to the PLA-PEG slightly increased the size of micelles, however the size remained small $(\sim 120 \mathrm{~nm})$ with a narrow distribution of particles (Fig. 1A). The RGD linking was evident as the overall zeta potential of micelles decreased from $\sim-28$ to $\sim-22 \mathrm{mV}$ (Fig. 1B). Both of the micelles showed high entrapment efficiency of $>90 \%$ and a markedly high loading capacity of $\sim 30 \%$. RGD substitution did not decrease the drug loading capacity of the micelles and presented self-assembled mono-dispersed spherical shaped particles.
In vitro drug release. The cumulative DTX release from both the DPP and RDPP micelles are presented in Fig. 1C. Free DTX exhibited a burst release profile with $60 \%$ of drug released within $24 \mathrm{~h}$. The drug-loaded micelles however, significantly controlled the release rate without any burst-release phenomenon $(\mathrm{P}<0.05)$. As expected, DPP and RDPP micelles showed similar release profiles throughout the study period. During a $24 \mathrm{~h}$ incubation $<20 \%$ of DTX was released, whilst $\sim 60 \%$ of the drug had been released by the end of seven days. Such a sustained release profile would be of significant importance for systemic delivery, or brain tumor targeting, where a substantial amount of drug is expected to locate in the intracellular environment. To investigate the release kinetics, the data were fitted to various mathematical models including zero order, first order and Higuchi. It was found that the micellar formulations were best fitted to the Higuchi model. These results indicated that diffusion was the governing mechanism of release. In addition, the Korsemeyer-Peppas equation indicated the presence of more than one mechanism of release (18).

Cytotoxicity assay. The cytotoxicity profiles of free DTX, DPP and RDPP when exposed to U87MG and 9L brain cancer cell lines for 24 and $48 \mathrm{~h}$ are shown in Fig. 2. All of the formulations showed time and concentration-dependent cytotoxicity on both of the cell lines. Marked differences were observed in the RDPP group, which exhibited significantly greater cytotoxicity as compared with the free drug and DPP groups $(\mathrm{P}<0.05)$. It was hypothesized that the pronounced cytotoxicity of RDPP may result from the enhanced internalization of the particles. The potent cytotoxic action of RGD-linked particles may increase the chances of brain cancer recovery. Conversely, 
A

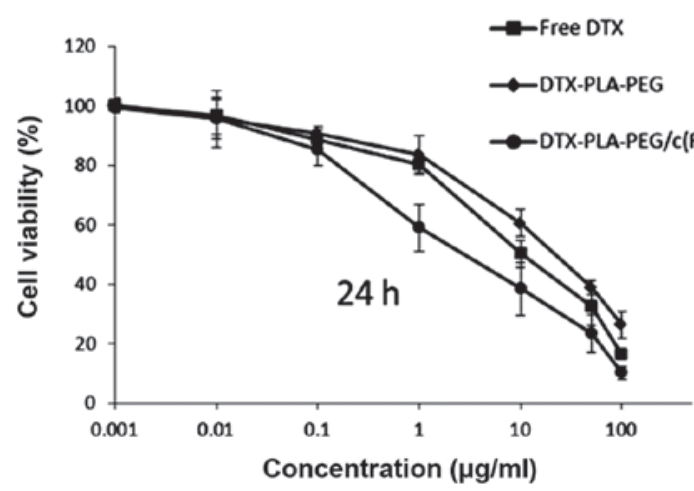

C

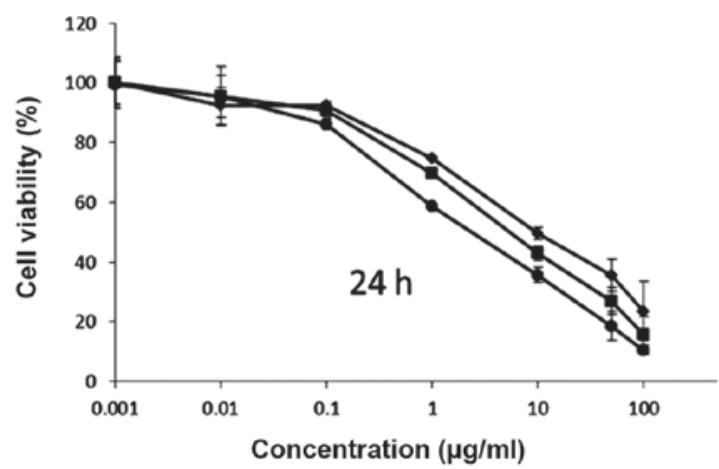

U87MG

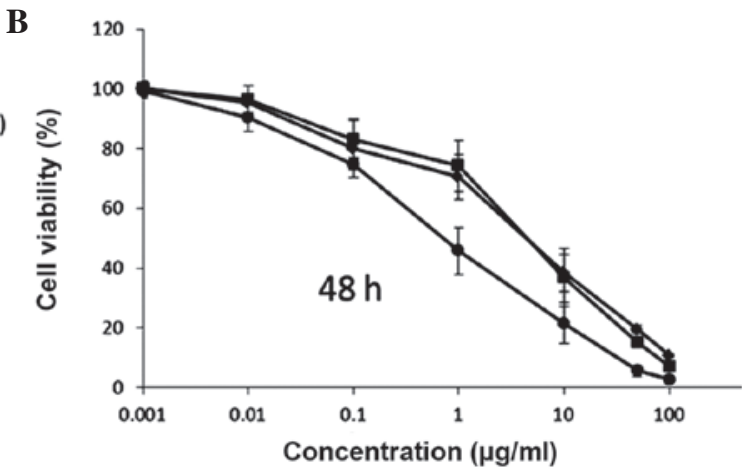

9L

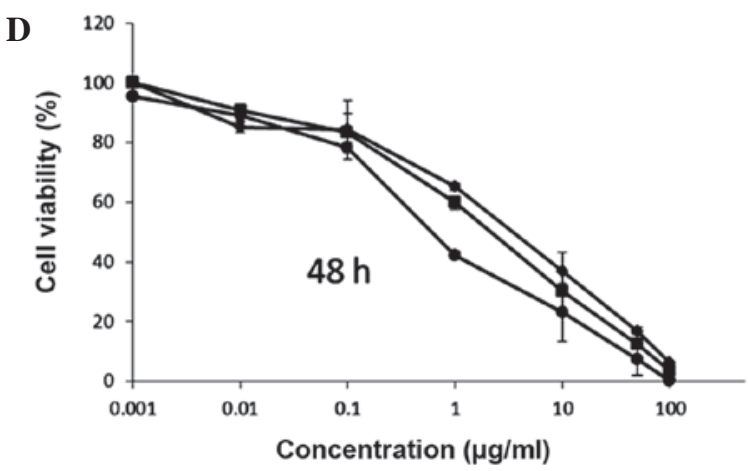

Figure 2. In vitro cytotoxic analysis of different concentrations of docetaxel (DTX) on (A and B) U87MG and (C and D) 9L glioblastoma cancer cell lines. The cell viability was determined by MTT assay. The cells were seeded at a density of 10,000 cells per/well and incubated for $24 \mathrm{~h}$ prior to the exposure of free DTX, docetaxel polylactic acid-polyethylene glycol (DTX-PLA-PEG) or DTX-PLA-PEG/cyclic (Arginine-Glycine-Aspartic acid-D-Tyrosine-Lysine) [DTX-PLA-PEG/c(RGDyK)] micelles for $24 \mathrm{~h}$ and $48 \mathrm{~h}$ at $37^{\circ} \mathrm{C}$.

A

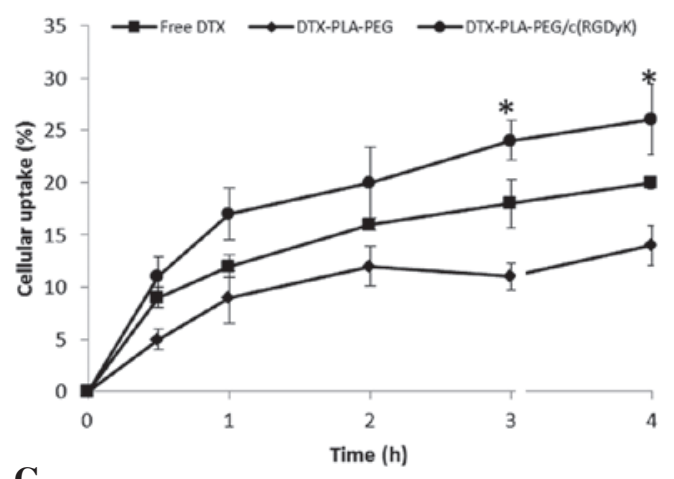

C

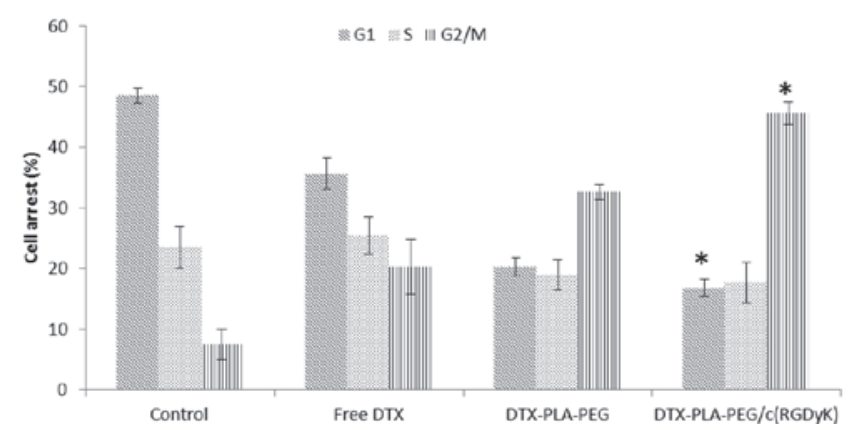

B

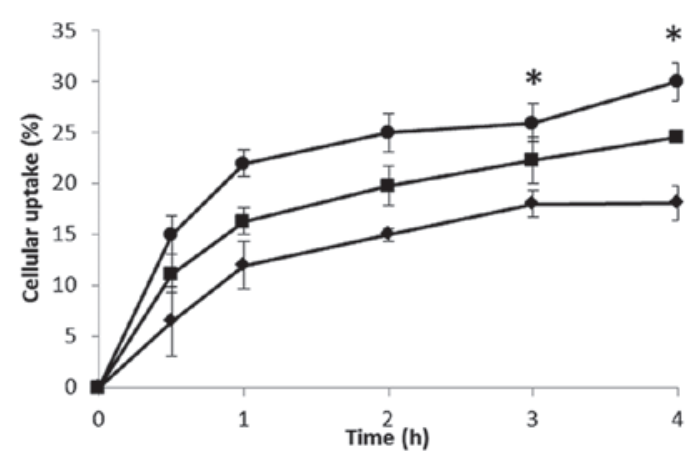

D

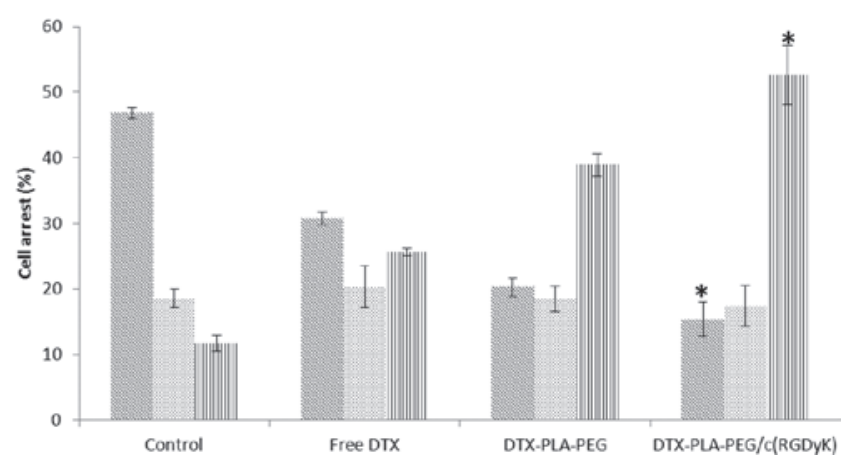

Figure 3. Cellular uptake efficiency of free docetaxel (DTX), docetaxel polylactic acid-polyethylene glycol (DTX-PLA-PEG) or DTX-PLA-PEG/cyclic (Arginine-Glycine-Aspartic acid-D-Tyrosine-Lysine) [DTX-PLA-PEG/c(RGDyK)] micelles in (A) U87MG and (B) 9L gliobastoma cancer cell lines. The cells were incubated with $25 \mu \mathrm{g} / \mathrm{ml}$ (DTX equivalent) for between $1-4 \mathrm{~h}$ and analyzed by high-throughput liquid chromatography. (C) U87MG and (D) 9L cells were treated with different formulations and cell cycle analysis was then performed using flow cytometry. "P $<0.05$, vs. free DTX. 
free DTX showed higher cytotoxicity as compared with DPP, which is consistent with a previously published report which stated that free drugs diffuse easily into the cell nucleus, whereas micellar drugs have to be detached from the NP prior to exhibiting any therapeutic action (19). The 9L cells were relatively more sensitive to all of the formulations, as compared with the U87MG cells. The difference in the cytotoxic effects on both of the cell lines may be attributed to the difference in genetic origin and indigenous biological behavior (20). In order to precisely quantify the effects of the individual therapeutic systems, the half maximal inhibitory concentration $\left(\mathrm{IC}_{50}\right)$ was evaluated. The $\mathrm{IC}_{50}$ values of free DTX, DPP and RDPP in U87MG were 8.56, 9.89, and $3.26 \mu \mathrm{g} / \mathrm{ml}$, respectively following $24 \mathrm{~h}$ incubation, while these values decreased to $2.18,2.38$, and $1.05 \mu \mathrm{g} / \mathrm{ml}$ following $48 \mathrm{~h}$ incubation. The respective values in 9L cells were $6.85,7.92$, and $2.54 \mu \mathrm{g} / \mathrm{ml}$ for the $24 \mathrm{~h}$ incubation and $1.68,1.74$, and $0.76 \mu \mathrm{g} / \mathrm{ml}$ for the $48 \mathrm{~h}$ incubation periods. These results suggest that the specific interactions of the targeting ligand and the enhanced cellular uptake of carriers during a longer incubation period may greatly increase the drug cytotoxicity. Therefore, actively targeted RDPP may be preferentially taken up by the cells through receptor-mediated endocytosis resulting in increased cytotoxicity, whereas non-targeted micelles may enter by normal passive uptake mechanisms only (21).

Cell uptake. The majority of delivery systems exert their therapeutic action following internalization into the cells. Therefore, the present study investigated the cellular uptake efficiency of U87MG and 9L cancer cells, that can augment the accumulation of drugs. The cellular uptake of both the free drug and the NPs increased in a time-dependent manner up to $4 \mathrm{~h}$ (Fig. 3a,b). Maximum internalization was completed by $1 \mathrm{~h}$, followed by a relatively slower uptake until the end of $4 \mathrm{~h}$. As expected, the RDPP group exhibited a significantly higher uptake, as compared with that of either the free drug or DPP groups $(\mathrm{P}<0.05)$. This may be due to the presence of the RGD moiety on the surface, which has a selective affinity towards the overexpressed $\alpha v \beta 3$ and $\alpha v \beta 5$ integrins on the cancer cell surface (22). It has previously been demonstrated that RGD-based complexes are internalized by a combination of various mechanisms; including clathrin and caveolae-mediated endocytosis, clathrin and caveolae-independent endocytosis and macropinocytosis $(23,24)$. The free DTX was continuously internalized in both of the cell lines, through passive diffusion. Specifically, 9L GBM cells showed relatively higher cellular uptake as compared with the U87MG cells. These data suggest that the RGD delivery system may be preferentially recognized by the integrin receptors present on the GBM cell surface, and may be internalized by various receptor and energy-dependent processes. Consequently, the active targeting moiety may be used to target the cancer cells upon intravenous injection and the higher uptake efficiency can rapidly remove the drug from the circulation.

Cell cycle analysis. The polymerization and depolymerization of microtubules has an important role in biological processes. DTX tightly binds microtubules, and promotes their stabilization, resulting in the mitotic arrest of cancer cells (25). It is well known that DTX induces cell cycle

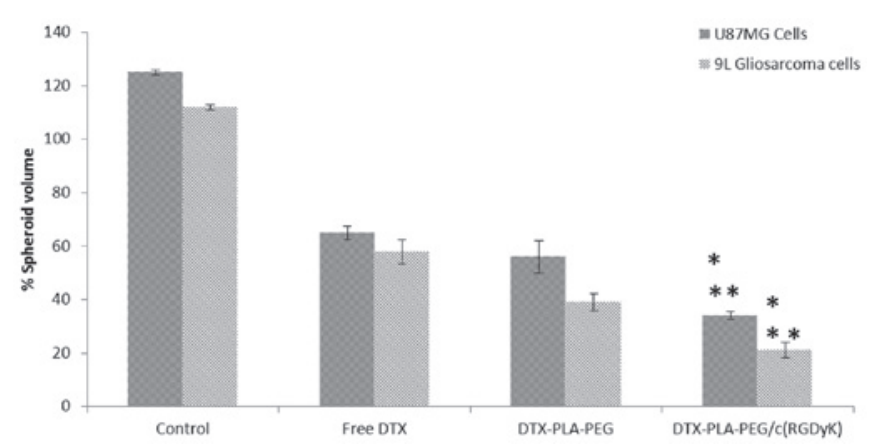

Figure 4. Change in glioma spheroid volume upon exposure to the different formulations: Free docetaxel, docetaxel polylactic acid-polyethylene glycol (DTX-PLA-PEG) or DTX-PLA-PEG/cyclic (Arginine-Glycine-Aspartic acid-D-Tyrosine-Lysine) [DTX-PLA-PEG/c(RGDyK)] micelles, at a dose of $1 \mu \mathrm{g} / \mathrm{ml}$, on U87MG and 9L gliobastoma cancer cell lines. ${ }^{*} \mathrm{P}<0.05$, vs. free DTX; ${ }^{* *} \mathrm{P}<0.01$, vs. control.

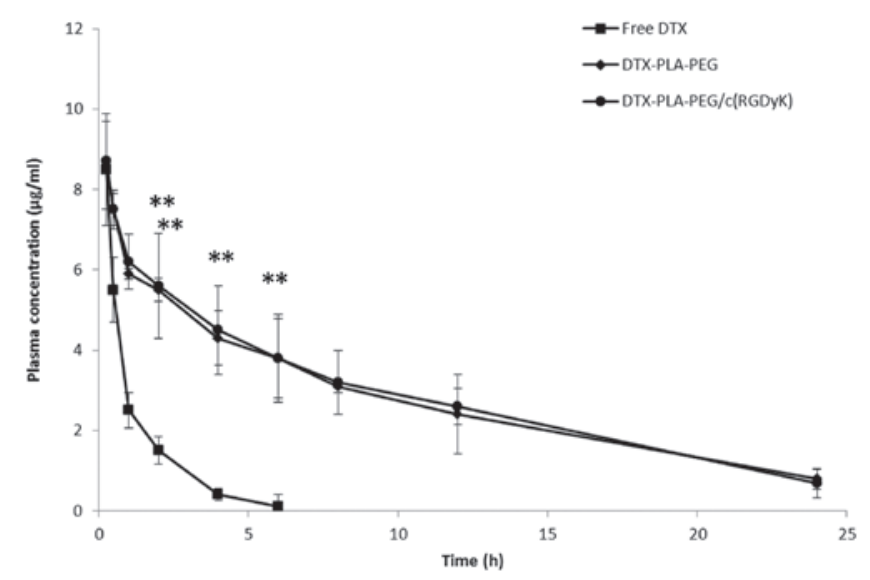

Figure 5. Plasma concentration-time profile of docetaxel (DTX) following a single dose intravenous administration of free docetaxel, docetaxel polylactic acid-polyethylene glycol (DTX-PLA-PEG) or DTX-PLA-PEG/cyclic (Arginine-Glycine-Aspartic acid-D-Tyrosine-Lysine) [DTX-PLA-PEG/ $\mathrm{c}(\mathrm{RGDyK})]$ micelles. The formulations were administered via a tail vein injection, at a dose of $10 \mathrm{mg} / \mathrm{kg}$. ${ }^{* *} \mathrm{P}<0.01$, vs. free DTX.

arrest through the impairment of mitosis and chromosomal damage, thereby inducing typical G2/M phase arrest (26). The cell cycle analyses of the cells treated with the different formulations are presented in Fig. 3C and D. The data shows the presence of the cell populations in the different phases upon treatment with the drug formulations. In the control group $\sim 50 \%$ cells were in the G1 phase, whereas only $\sim 7 \%$ cells were in the $\mathrm{G} 2 / \mathrm{M}$ phase. In the RDPP group $~ 45 \%$ cells were shown to be in G2/M phase, as compared with $\sim 30$ and $\sim 20 \%$ for the DPP and free DTX groups in U87MG cells, respectively. A similar trend was observed in 9L cells, however the proportion of cells in G2/M phase was relatively higher as compared with U87MG cells. The superior cell arresting effects of the RDK-linked formulations was consistent with the higher cellular uptake and cytotoxic potential, demonstrated in both of the cancer cell lines. It was hypothesized that RDPP may promote the stabilization of microtubules and differentiation of cancer cells. This may result in the downregulation of tubulin, leading to the impairment of mitotic spindle formation and eventually cell cycle arrest. 

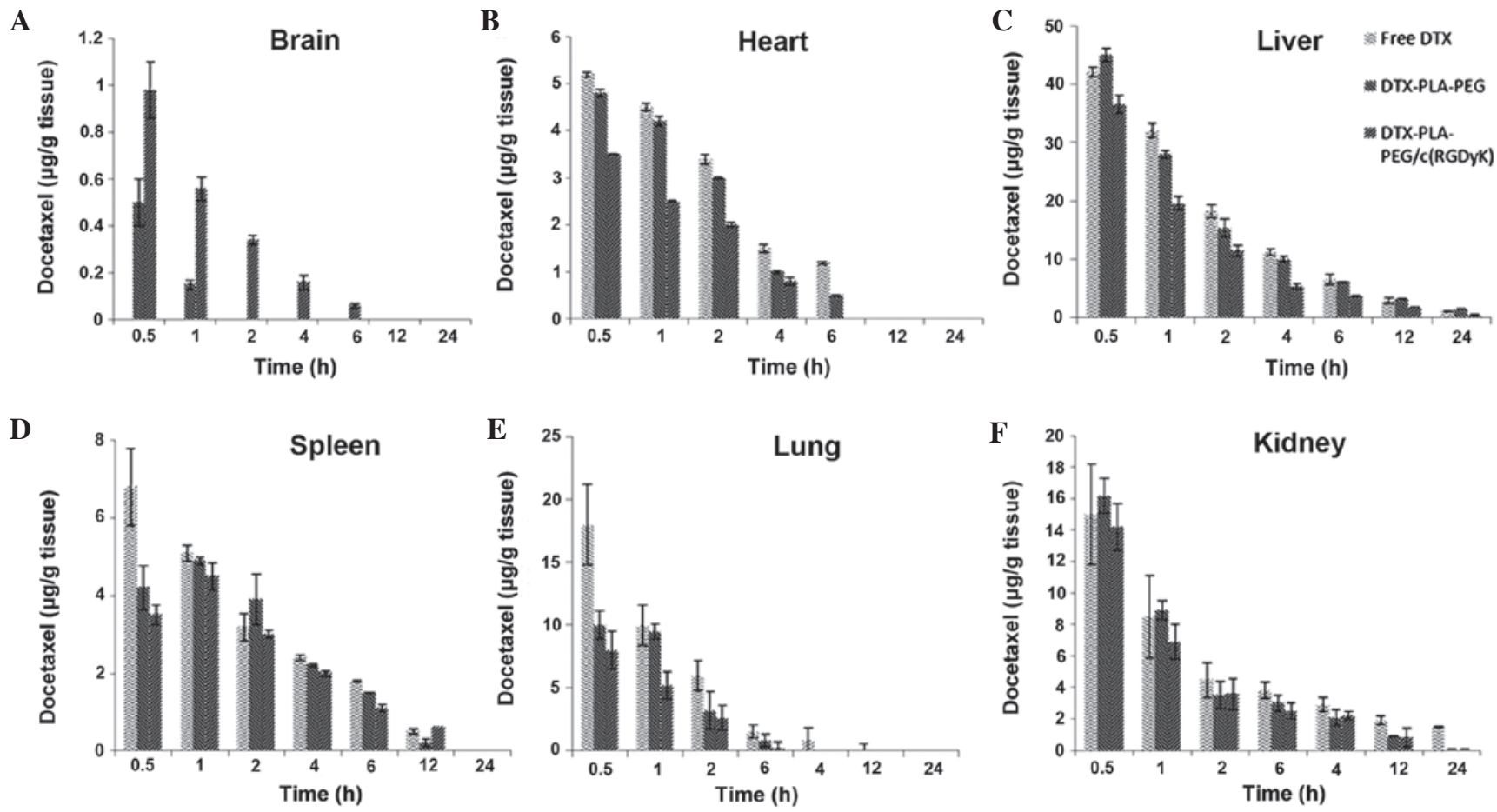

Figure 6. Biodistribution of free docetaxel (DTX), docetaxel polylactic acid-polyethylene glycol (DTX-PLA-PEG) or DTX-PLA-PEG/cyclic (Arginine-Glycine-Aspartic acid-D-Tyrosine-Lysine) [DTX-PLA-PEG /c(RGDyK)] micelles in U87MG tumor-bearing nude xenograft mice in different organs following intravenous administration of the different formulations. (A) Brain, (B) heart, (C) liver, (D) spleen, (E) lung, (F) kidney. The mice were sacrificed at $0.5,1,2,4,6,12$, and $24 \mathrm{~h}$ post-injection.
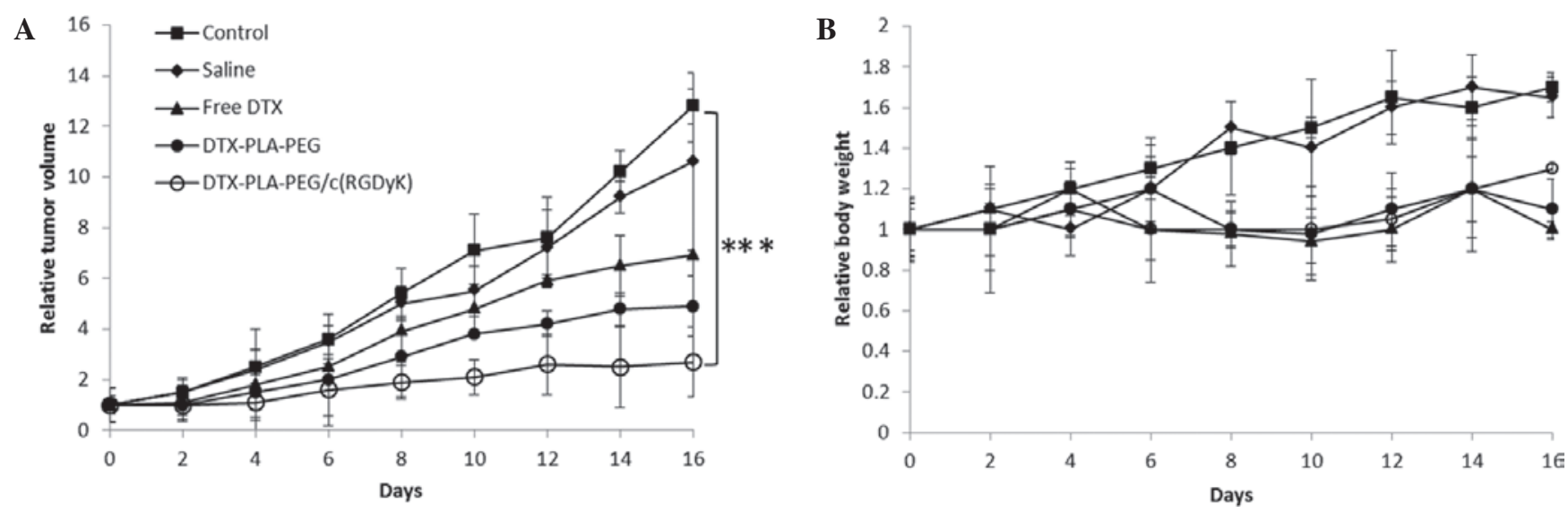

Figure 7. In vivo antitumor efficacy upon treatment with free docetaxel (DTX), docetaxel polylactic acid-polyethylene glycol (DTX-PLA-PEG) or DTX-PLA-PEG/cyclic (Arginine-Glycine-Aspartic acid-D-Tyrosine-Lysine) [DTX-PLA-PEG/c(RGDyK)] micelles in U87MG tumor-bearing nude xenograft mice. (A) The change in tumor volume and (B) the variation in body weight as a function of time. The formulations were administered at a dose of $5 \mathrm{mg} / \mathrm{kg}$ by tail vein injection four times over an interval of three days. ${ }^{* * *} \mathrm{P}<0.01$, vs. control.

Analysis of in vitro spheroid inhibition. The inhibitory effects of free DTX, DPP and RDPP on the growth of U87MG and 9L spheroids are presented in Fig. 4. Spheroids in the untreated group grew rapidly and attained a maximum volume of $\sim 125$ and $\sim 110 \%$ for U87MG and 9L cells, respectively. In contrast, the free and micellar drugs significantly inhibited the growth of the spheroids $(\mathrm{P}<0.05)$. The spheroids appeared distorted, disintegrated, shrunken and had lost their typical three dimensional structures. RDPP treatment resulted in a $>80 \%$ reduction in spheroidal volume, indicating its superior inhibitory effects. Free DTX could directly kill the cells on the surface, however transport across the spheroid proved difficult.
In contrast, hydrophobic core-containing micelles exhibited a preferential uptake. RGD-mediated cellular uptake may further increase the drug concentration within the spheroids. The inhibitory effect was in the order of RDPP $>$ DPP $>$ DTX. The stronger inhibitory effects of RDPP may be due to its better permeability within the cells, resulting in superior in vitro therapeutic effects (27).

Evaluation of pharmacokinetics. The plasma concentration-time profiles of DTX, following a single dose intravenous administration of free DTX, DPP or RDPP, are presented in Fig. 5. Free DTX was immediately removed from the blood 
circulation and reached the detection limit within $6 \mathrm{~h}$ of the study period. In contrast, the micellar formulations significantly enhanced the circulatory performance of DTX $(\mathrm{P}<0.05)$. Substitution of PEG with the RGD block did not hamper the long circulation profile of RDPP, and a substantial concentration of DTX was detected throughout the study period (24 h). RDPP showed a 3-4 fold higher area under curve $(36.85 \pm 4.52$ $\mathrm{h} \mu \mathrm{g} / \mathrm{ml})$ as compared with the free DTX $(7.96 \pm 2.62 \mathrm{~h} \mu \mathrm{g} / \mathrm{ml})$. Consequently, RDPP showed a significantly higher terminal half-life of $4.57 \mathrm{~h}$, as compared with $0.91 \mathrm{~h}$ of the free drug. The longer circulation profile of RDPP may promote the likelihood of BBB permeation and higher tumor targeting ability.

Biodistribution study. The concentration of DTX present in the mouse organs, including the brain, heart, spleen, liver, kidney, and lungs were investigated at different time points. The study was performed following a single dose intravenous injection of each formulation via the tail vein. The results clearly showed that RDPP was able to deliver the DTX into the brain at a significantly higher level as compared with the non-targeted DPP micelles and the free drug $(\mathrm{P}<0.05$; Fig. 6A). RDPP exhibited $\sim 1 \mu \mathrm{g}$ DTX/g tissue after $30 \mathrm{~min}$; this level gradually decreased up to $6 \mathrm{~h}$, after which no drug was detected. Conversely, DPP showed $\sim 0.5 \mu \mathrm{g}$ DTX/g tissue at $30 \mathrm{~min}$, which immediately decreased to the lowest level at $1 \mathrm{~h}$, after which no drug was detected. No drug was detected in the free DTX group. The significantly higher concentration $(\mathrm{P}<0.05)$ observed in the RDPP group was consistent with its ability to interact with the cancer cells via receptor mediated endocytosis, whereas DPP could not influence the cellular uptake beyond a certain limit. Furthermore, the $\mathrm{BBB}$ is randomly disrupted in brain tumors, along with the components of the tumor blood vessels including the basement membrane, pericytes and the endothelial cells which provide entry for the nanosized particles (28). This further proves that passive targeting is inefficient in maintaining adequate concentrations of drugs in brain cancer cells. RGD-linking, however, along with an increased circulative ability, markedly enhanced the drug concentration levels. In the heart, liver and spleen, RDPP accumulated at relatively low levels, suggesting that drug-related systemic cytotoxicity may be effectively avoided (Fig. 6B-F). The reduced presence of the drug in the lung, in response to treatment with either of the micellar formulations, may be due to its nanosize, which may avoid enhanced uptake by the lung tissue.

Antitumor efficacy. A U87MG-bearing tumor model was developed and treated with the various formulations. As shown in Fig. 7, RDPP significantly inhibited the growth of glioblastoma tumors, as compared with that of the control and free drug treated groups of mice $(\mathrm{P}<0.05)$. The tumor volume remained the same until day four, however after that, significant differences were observed between the different groups. Tumors in the saline and control groups grew rapidly and attained maximum size. The marked antitumor efficacy of the RGD-linked micelles was attributed to the specific binding with the highly angiogenic neovasculature of subcutaneously grown GBM cells. Consistent with the long circulation profile and the drug accumulation in the specific tumors, enhanced tumor regression may be anticipated. Similar results have been reported by previous studies which demonstrated the improved antitumor effects for RGD-linked delivery systems $(29,30)$.

The body weight fluctuations of the mice were assessed in terms of in vivo systemic toxicity of the drug and delivery system. Body weight of the individual mice was noted throughout the study period alongside the tumor volume measurement. The mice administered with saline and the control group experienced a gain in body weight, attributed to an increased tumor volume. In the DPP and RDPP administered groups, no loss of body weight was observed, indicating a lack of any serious drug-related toxicity.

Thus far, glioblastoma, which represents one of the deadliest forms of cancer, is unresponsive to chemotherapy mainly due to the poor penetrability of therapeutics across the BBB and BBTB. Therefore, the aim of the present study was to design a delivery system, appended to a targeting ligand, that could overcome these physiological barriers and deliver the anticancer drugs into the glioma cells. GBM is generally characterized by angiogenesis and over-expression of the integrin $\alpha v \beta 3$ receptor on the surface of the tumor neovasculature. In the present study c(RGDyK) peptide was selected as a specific targeting agent to the integrin receptors. The results clearly showed that RDPP could effectively bind the $\alpha v \beta 3$ receptor, facilitating the cellular uptake and inreasing the cytotoxicity of the drug. Overexpression of the $\alpha v \beta 3$ receptor resulted in an enhanced accumulation of RDPP in the brain tumor cells in vivo, whilst non-targeted micelles had limited access. RGD-linked micelles showed pronounced tumor regression with an excellent safety profile. Therefore, the results clearly revealed the superior antitumor efficacy of c(RGDyK)/DTX-PLA-PEG in an experimental brain tumor model.

In conclusion, DPP and RDPP were successfully prepared and subjected to various in vitro and in vivo characterizations. RDPP showed enhanced accumulation in U87MG and 9L glioblastoma cell lines, through various endocytic pathways, and displayed pronounced cytotoxic effects in the cell lines with markedly lower $\mathrm{IC}_{50}$ values. Furthermore, RDPP showed a typical G2/M phase arrest and stronger microtubule stabilizing effects. Consequently, high accumulation and distribution of RDPP resulted in tremendous growth inhibition of glioma spheroids, as compared with the non-targeted DPP and free drug. The ability of RDPP to bind the $\alpha v \beta 3$ integrin receptor resulted in a high accumulation of DTX in the brain glioblastoma cells, which in turn may be due to the specific interaction of micelles, small particle size and an extended blood circulation profile. The enhanced tumor regression in the U87MG tumor-bearing mice further confirmed the superior anti-glioblastoma efficacy of RDPP. The findings of the present study suggest that $\mathrm{c}(\mathrm{RGDyK})$ linked to delivery carriers may be a potential strategy for the treatment of advanced brain cancer.

\section{Acknowledgements}

The authors would like to thank Dr. Shiam for proof reading the manuscript.

\section{References}

1. Jain RK, di Tomaso E, Duda DG, et al: Angiogenesis in brain tumors. Nat Rev Neurosci 8: 610-622, 2007. 
2. Ong BY, Ranganath SH, Lee LY, et al: Paclitaxel delivery from PLGA foams for controlled release in post-surgical chemotherapy against glioblastoma multiforme. Biomaterials 30: 3189-3196, 2009.

3. Wen PY and Kesari S: Malignant Gliomas in Adults. N Engl J Med 359: 492-507, 2008.

4. Jones TS and Holland EC: Standard of care therapy for malignant glioma and its effect on tumor and stromal cells. Oncogene 31: 1995-2006, 2012.

5. Rich JN and Bigner DD: Development of novel targeted therapies in the treatment of malignant glioma. Nat Rev Drug Discov 3: 430-446, 2004.

6. Ningaraj NS: Drug delivery to brain tumours: challenges and progress. Expert Opin Drug Deliv 3: 499-509, 2006.

7. Sarin H: Recent progress towards development of effective systemic chemotherapy for the treatment of malignant brain tumors. J Transl Med 7: 77, 2009.

8. Xin H, Sha X, Jiang X, Zhang W, Chen L and Fang X: Anti-glioblastoma efficacy and safety of paclitaxel-loading Angiopep-conjugated dual targeting PEG-PCL nanoparticles. Biomaterials 33: 8167-8176, 2012.

9. $\mathrm{Gu} \mathrm{G}, \mathrm{Xia} \mathrm{H}, \mathrm{Hu} \mathrm{Q}$ et al: PEG-co-PCL nanoparticles modified with MMP-2/9 activatable low molecular weight protamine for enhanced targeted glioblastoma therapy. Biomaterials 34:196-208, 2013

10. Peer D, Karp JM, Hong S, Farokhzad OC, Margalit R and Langer R: Nanocarriers as an emerging platform for cancer therapy. Nat Nanotechnol 2: 751-760, 2007.

11. Danhier F, Le Breton AL and Préat V: RGD-based strategies to target alpha(v) beta(3) integrin in cancer therapy and diagnosis. Mol Pharm 9: 2961-2973, 2012.

12. Schottelius M, Laufer B, Kessler H and Wester HJ: Ligands for mapping alphavbeta3-integrin expression in vivo. Acc Chem Res 42: 969-980, 2009.

13. Chen X, Park R, Shahinian AH, et al: 18F-labeled RGD peptide: initial evaluation for imaging brain tumor angiogenesis. Nucl Med Biol 31: 179-189, 2004.

14. Chen X, Hou Y, Tohme M, et al: Pegylated Arg-Gly-Asp peptide: $64 \mathrm{Cu}$ labeling and PET imaging of brain tumor alphavbeta3-integrin expression. J Nucl Med 45: 1776-1783, 2004.

15. Gajbhiye V and Jain NK: The treatment of Glioblastoma Xenografts by surfactant conjugated dendritic nanoconjugates. Biomaterials 32: 6213-6225, 2011.

16. Nasongkla N, Bey E, Ren J et al: Multifunctional polymeric micelles as cancer-targeted, MRI-ultrasensitive drug delivery systems, Nano Lett 6: 2427-2430, 2006.

17. Zhan C, Gu B, Xie C, Li J, Liu Y and Lu W: Cyclic RGD conjugated poly(ethylene glycol)-co-poly(lactic acid) micelle enhances paclitaxel anti-glioblastoma effect. J Control Release 143: 13620-142, 2010.
18. Ramasamy T, Khandasami US, Ruttala $\mathrm{H}$ and Shanmugam S: Development of solid lipid nanoparticles enriched hydrogels for topical delivery of anti-fungal agent. Macromol Res 20: 682-692, 2012.

19. Carrion C, de Madariaga MA and Domingo JC: In vitro cytotoxic study of immunoliposomal doxorubicin targeted to human CD34(+) leukemic cells. Life Sci 75: 313-328, 2004.

20. Carvalho JC, Perazzo FF, Machado L and Bereau D: Biologic activity and biotechnological development of natural products. BioMed Res Int 2013: 971745, 2013.

21. Gupta B and Torchilin VP: Monoclonal antibody 2C5-modified doxorubicin-loaded liposomes with significantly enhanced therapeutic activity against intracranial human brain U-87 MG tumor xenografts in nude mice. Cancer Immunol Immunother 56 1215-1223, 2007.

22. Liu J and Shapiro JI: Endocytosis and signal transduction: basic science update. Biol Res Nurs 5: 117-128, 2003

23. Jiang X, Sha X, Xin H et al: Self-aggregated pegylated poly (trimethylene carbonate) nanoparticles decorated with c(RGDyK) peptide for targeted paclitaxel delivery to integrin-rich tumors. Biomaterials 32: 9457-9469, 2011.

24. Jiang X, Sha X, Xin H et al: Integrin-facilitated transcytosis for enhanced penetration of advanced gliomas by poly(trimethylene carbonate)-based nanoparticles encapsulating paclitaxel. Biomaterials 34: 2969-2979, 2013.

25. Downing KH and Nogales E: Tubulin and microtubule structure. Curr Opin Cell Biol 10: 16-22, 1998.

26. Miller ML and Ojima I: Chemistry and chemical biology of taxane anticancer agents. Chem Rec 1: 195-211, 2001.

27. Gao H, Yang Z, Zhang S, et al: Glioma-homing peptide with a cell-penetrating effect for targeting delivery with enhanced glioma localization, penetration and suppression of glioma growth. J Control Release 172: 921-928, 2013

28. Kanazawa T, Taki H, Tanaka K, Takashima Y and Okada H: Cell-penetrating peptide-modified block copolymer micelles promote direct brain delivery via intranasal administration. Pharm Res 28: 2130-2139, 2011.

29. Zhao H, Wang JC, Sun QS, et al: RGD-based strategies for improving antitumor activity of paclitaxel-loaded liposomes in nude mice xenografted with human ovarian cancer. J Drug Target 17: 10-18, 2009.

30. Saad M, Garbuzenko OB, Ber E, et al: Receptor targeted polymers, dendrimers, liposomes: which nanocarrier is the most efficient for tumor-specific treatment and imaging? J Control Release 130: 107-114, 2008. 